\title{
PROTECTION OF ROAD INFRASTRUCTURE - DYNAMIC WEIGHING OF VEHICLES
}

\author{
Peter Hronský \\ Výskumný ústav dopravný, a.s., Vel'ký diel 3323, 01008 Žilina, Slovenská republika, hronsky@vud.sk
}

Keywords: weighing in motion, legislation, system requirements

Abstract: The reason of the construction of the official measurement of axle loads through dynamic weighing of the trucks and buses on the road is to prevent damage to roads and shortening their lifespan and operational capability due to the crossing of axle loads on trucks' and buses' limits with a gross weight of 3.5 tons. Article provides an analysis of legislation for the establishment of a system for driving vehicles weighing (weighing in motion - WIM) at high speed, the identification of essential requirements and the issue of strict liability in the field. Description is also considering the usage of the part of the technical infrastructure of the existing toll system.

\section{Introduction}

The analysis reveals, the weighing issue itself in applicable legislation concerns immediately only the SR Act. 135/1961 Coll. on roads (Road Act), as amended (the "Road Law"), which contains provisions governing the issue of weighting, security establishment, the establishment and operation of scales and weighing treatment.

The introduction of changes to the official measurement will entail Road Act, under which it will be necessary to define exactly the terms low-speed and highspeed weighing, conditions for their establishment and operation as well as the rights and obligations of the various parties. The recommended solution is to include the treatment of the basic conditions for the establishment of weights and weighing in road law, respectively in the new draft Road Act and any details left to subordinate legislation through empowering provisions in the law.

\section{The official gauges}

Definition of official instruments, method of metrological control, technical and metrological requirements are regulated in Decree of the Office for Standards, Metrology and Testing of the Slovak Republic no. $210 / 2000$ Z. z. on measuring instruments and metrological control, which Act no. 142/2000 Coll. on Metrology and on amendments to certain acts (hereinafter the "Law on metrology"). The legislation of the Metrology Act is sufficient, with the necessary legislative changes encompassed only within the framework of implementing regulations. The accuracy of the scales cannot be lower (worse) than maximal permissible overload (currently 3\%). Therefore, current standards do not allow the use of high-speed weighing on the market with an accuracy of $5 \%$.

To enable high-performance measurements should MDVRR SR:
1. insist on high-speed precision weighing $5 \%$, or

2. Increase max. congestion of vehicles permitted to $5 \%$.

\section{System Requirements}

In general, the data obtained from WIM system can be stated that:

- It is large amount of data,

- Records for each type of vehicle may be of different lengths.

Requirements for data management can be formulated as follows:

- Supporting for multiple formats of data files,

- Rigorous quality assurance process,

- Data is recorded by way ,vehicle after vehicle”,

- Aggregated data for unified presentation system (e.g. GIS use)

- Vehicle relational database (tables of the vehicles and their axles)

- The distribution of the database by location (location)

- Distributed "layers" for extensibility: Data - application - presentation,

- Stored data is continuously online,

- Customizable solution - the ability to add additional users, sites and hardware.

For the purposes of search, creation, analysis and statistics the system should provide for data filtering by various criteria, e.g.:

- vehicles' size,

- vehicles' class,

- (Dis) approval with mass data.

- (Dis) approval with the permissible size,

- Locality,

- Time

- Vehicles' registration,

- Repeatedly overloaded vehicles and their operator 
- Declared (approved) excessive traffic,

- Vehicles with the exception,

- Specific vehicles (dangerous goods).

Requirements for road equipment:

The system must ensure single threshold weighing and proper operation in a wide range of vehicle speeds and other boundary conditions. Weighing shall consist of weighing scales in each lane. Weight sensors should cover the entire width of the lane. WIM system consists of the following components:

- Wheel weights record data on weight for each track width (right and left axle load); the weighing data to be uniform over the whole width scales,

- The control unit (road unit, RSE - Road Side Equipment), which is installed in a cabinet, comprises equipment and software for the calculation, storage and transmission of all the specified data to the host computer (WIM in centre); the control unit further comprises:

- the tools to access control system (e.g. keyboard and monitor or computer means to secure access, possibly hardware key) delivered as a packaged unit,

- the unit has to work with an external source of electrical power with battery backup to provide continuous power for at least 1 hour. (Ed .: in case of power through the toll enforcement gates should be based on the specifications of the toll system, whether it be addressed backup in the control unit or power supply as a whole is left to the enforcement gate of the toll system as power consumption of the weight control unit comparing to the gate one is a little),

- the modem compatible with the communication network and with the modem in the WIM centre is only necessary when communication does not take place within a supervisory communication network subsystem toll system

- Protection against disturbances (light and electricity) is necessary so far as it is not provided by the other way (e.g. lighting of camera and interference power of the power source need not be so long as such matters as a surveillance of the goal).

Functional system requirements can be formulated as follows:

- WIM system must be able to assess vehicles and vehicle combinations for a total of 12 axles and must be automatically determined for each vehicle in the lane:

- the load on each axle with a load of left and right wheel speed, axle spacing and length of the vehicle,

- the classification of the vehicles - WIM system must provide a resolution of 15 classes of vehicles, with the first 13 classes are defined exactly 14 class presents special vehicles, 15 class is allocated to other vehicles which can not be classified in the first 14 classes; classification criteria are programmable as defined by the user,
- an error of measurement - the measurement should be evaluated as invalid if:

- for the measurement conditions have not been respected certification,

- left and right wheel load of any axle varies by $40 \%$ or more,

- any wheel load of that axle exceeds $8.9 \mathrm{kN}$.

Both values are programmable by the operator. No vehicle, which was carried out for erroneous measurement shall not be considered to be "weighed" vehicle, but may be classified and accounted for and all data are stored in the vehicle record.

o the detection of the offense - for the vehicle's weight WIM system must to determine which (if any) axles, axle or axle group weight exceeds the limits set out in "table being exceeded"' the values in the table are generally pre-set limits, however, the weight can be defined by the user,

- Control unit quantify and retain all the necessary data to storage media - storage medium of the WIM station must have a capacity to store at least 14 daily records of the number of vehicles and individual vehicle data; the storage medium should be of a type which will not cause a state of data e.g. due to interruption of power supply. The controller must continuously compute and store data for all vehicles passing through the system. WIM controller must keep the following data:

- an hour numbers of vehicles by lane by class and by speed intervals for each 24-hour period,

o the single record of the vehicle shall contain at least the following information:

time and date,

registration number of the record created,

lane, direction of travel,

vehicle registration number,

vehicle speed,

vehicle classification (category),

weight of each wheel or dual wheel under the vehicle and under the axles, axle and total weight,

axles, spacing (wheelbase) between each of the following numbered axle,

the overall length of each vehicle or assembly, time of separation from the previous vehicle,

the code of the offense in the field of overrun by mass,

the code for erroneous measuring / weighing photo document,

additional information (e.g. the outside temperature if necessary. Other meteorological data, recognized ADR tables, etc.).

- Data must be calculated and formatted such that all data can be accessed, and all required entries generated by using application software WIM system,

- All WIM control units must operate correctly at atmospheric temperature range of -40 to $+70^{\circ} \mathrm{C}$. 
PROTECTION OF ROAD INFRASTRUCTURE - DYNAMIC WEIGHING OF VEHICLES

Peter Hronský

- WIM control unit must also have communication capabilities that enable the personnel outside the WIM to keep track of operation at WIM site and allow data transmission to the WIM centre (remote access); WIM controller must also be able to join the staff present in the station for the purpose of diagnostics and downloading the data.

Other functional and technical requirements

- the fulfilment of metrological requirements,

- WIM stations are without the physical presence of support staff

- checking the compliance with the limit conditions to ensure the accuracy of weighing (i.e. whether it is generated by "certified" measurements)

- sorting and filtering data,

- the creation of analytical outputs.

- the creation of protocols in different languages,

- comparison the control data towards the current database of the allowed excessive rides,

- comparison of the data towards current databases of vehicles excepted,

- the generation of statistics on WIM area not connected directly with WIM (weather conditions, vehicle speed, traffic volume, the proportion of vehicles by category, ...)

- secured communication outside WIM centre (i.e. from the WIM centre towards the WIM stations, external users),

- the user-friendly interface system

- access to data according to defined permissions,

- the backup WIM database data in a data warehouse physical located outside WIM centre,

- the data logging of WIM operation site and its continuous mapping into WIM centre

- the logging of WIM operation centre

- system provides in a real time the overview of the functionality of the system and its components.

Note .: if dynamic weighing will be implemented (i.e., detection of overload) only for trucks and buses (other parameters can be provided for all registered vehicles), it is possible some aspects (incorrect measurement, detection of the offense, vehicle record) to be evaluated after fulfilling the conditions of minimum load on the front axle wheels (e.g. $15.6 \mathrm{kN}$ ).

Strict liability - legislative amendment

Strict liability means the duty of the holder of the vehicle to ensure compliance with the rules of the road selected by the driver to whom the vehicle is entrusted. For an offense committed with the vehicle will be responsible just the holder of the vehicle. The new responsibilities of the holders of the vehicle are valid from July 1st. 2012.

The provision of $\S 6$ a of the Act no. 8/2009 on Road traffic in the present Act introduces so called other administrative offense that may be committed by the holder of the vehicle.

If it is undoubtedly determined that an administrative offense has been by the car committed by specific holder, the Police authority without further action immediately issue an order imposing a fine for an administrative offense (the "Warrant"), whose content requirements will apply accordingly $\S 47$ of the Administrative Code.

\section{Solution after detection of overloaded vehicles}

The official measurement of the dynamic axle load and total weight of motor vehicles and buses at high speed with strict liability allows to immediately punish the offender by imposing a fine. The fine will be imposed on the owner of a motor vehicle that was evaluated as overloaded by high-speed dynamic weighing axle loads and total weight.

For vehicles that leave the Community's external border (SK / UA) will be necessary before the exit from the Slovak Republic to pay the fine through the Department of Border Police PPZ SR and then release the vehicle to the customs clearance.

Motor vehicles detected by a high-speed dynamic measurement of axle load and total weight of vehicles assessed as overloaded and facing the inner border of the Slovak Republic and the Community is necessary to address the fine imposed by strict liability and recover it before leaving territory of the Slovak Republic Department at toll police PPZ SR.

Passenger cars which are registered in the Slovak Republic must be addressed after finding overload condition of a motor vehicle through the Institute of strict liability.

Particular attention will be paid to motor vehicles which very significantly excess overloading the axle load or total weight. These should be addressed with a system lowspeed weighing or official measurement of the static axle load and total weight of motor vehicles using:

1. vehicle immobilisation,

2. transferring a motor vehicle escorted to a designated location because of transhipment and solutions recourse,

3. proposal for the closure of the carrier or to exclude the possibility of entry of a motor vehicle to the Slovak Republic.

\section{References}

[1] MIKULA, J., HRONSKÝ, P., HRUDKAY, K.: Protection of road infrastructure - dynamic weighing of vehicles . In: Final Report of the project, Žilina, 08/31/2012 . (Original in Slovak) 


\section{Acknowledgement}

The article was elaborated during the implementation of projects Research Centre of the University of Žilina -

ITMS 26220220183, funded within the Operational

Programme Research and Development.

\section{Review process}

Single-blind peer reviewed process by two reviewers. 